\title{
Development of Low Noise THz SIS Mixer Using an Array of Nb/Al-AlN/NbTiN Junctions
}

\author{
A. Karpov, D. Miller, J. A. Stern, B. Bumble, H. G. LeDuc, and J. Zmuidzinas
}

\begin{abstract}
We report the development of a low noise and broadband SIS mixer aimed for $1 \mathrm{THz}$ channel of the Caltech Airborne Submillimeter Interstellar Medium Investigations Receiver (CASIMIR), designed for the Stratospheric Observatory for Infrared Astronomy, (SOFIA).

The mixer uses an array of two $0.24 \mu \mathrm{m}^{2} \mathrm{Nb} / \mathrm{Al}$-AIN/NbTiN SIS junctions with the critical current density of $30-50 \mathrm{kA} / \mathrm{cm}^{2}$. An on-chip double slot planar antenna couples the mixer circuit with the telescope beam. The mixer matching circuit is made with $\mathrm{Nb}$ and gold films. The mixer IF circuit is designed to cover $4-8 \mathrm{GHz}$ band.

A test receiver with the new mixer has a low noise operation in 0.87-1.12 THz band. The minimum receiver noise measured in our experiment is $353 \mathrm{~K}(\mathrm{Y}=1.50)$. The receiver noise corrected for the loss in the LO injection beam splitter is $250 \mathrm{~K}$. The combination of a broad operation band of about $250 \mathrm{GHz}$ with a low receiver noise makes the new mixer a useful element for application at SOFIA.
\end{abstract}

Index Terms-Niobium alloys, radio astronomy, submillimeter wave receivers, superconductor-insulator-superconductor mixers, THz technology.

\section{INTRODUCTION}

$\mathbf{T}$ HE Earth atmosphere is nearly opaque at the Terahertz frequencies and one has to use the orbital or sub orbital platforms for astronomical observations in $\mathrm{THz}$ band. The Stratospheric Observatory for Infrared Astronomy (SOFIA) [1] is an example of such sub orbital platform. The observatory is based at a 747 Boeing flying a 2.5 meter telescope at an altitude up to $14 \mathrm{~km}$. A high cost of SOFIA operation and a limited observation time is making the sensitivity of the detectors, and thus the speed of the detection, a vital priority in this astronomical project.

Below we discuss the work on a mixer for heterodyne Caltech Airborne Submillimeter Interstellar Medium Investigations Receiver (CASIMIR) [2], designed for the Stratospheric Observatory for Infrared Astronomy, (SOFIA). It is a spectrometer dedicated to a high resolution spectroscopy of molecular lines at SOFIA.

Manuscript received August 27, 2008. First published June 30, 2009; current version published July 10, 2009. This work was supported by USRA SOFIA instrument development program.

A. Karpov, D. Miller and J. Zmuidzinas are with the Physics Department, California Institute of Technology, Pasadena, CA 91125 USA (e-mail: karpov@submm.caleh.edu).

J. A. Stern, B. Bumble and H. G. LeDuc are with MDL, Jet Propulsion Laboratory, California Institute of Technology, Pasadena, CA 91109 USA.

Color versions of one or more of the figures in this paper are available online at http://ieeexplore.ieee.org.

Digital Object Identifier 10.1109/TASC.2009.2017913

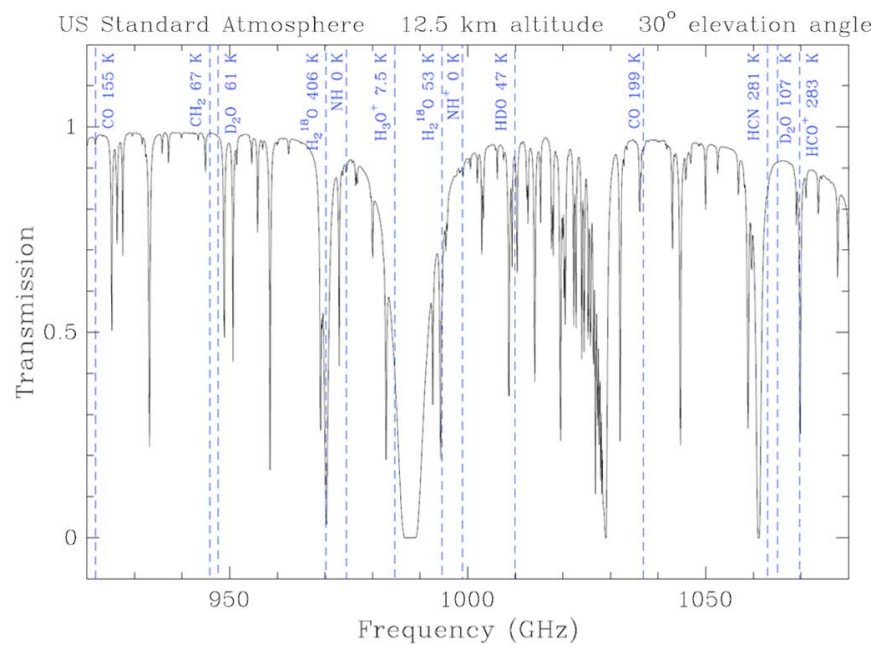

Fig. 1. The atmospheric transmission in $0.9-1.1 \mathrm{THz}$ band of SOFIA observatory flying at $12 \mathrm{~km}$ altitude with telescope at 30 degree elevation angle calculated using ATM [3]. A number of interesting molecular lines is located at the frequencies with nearly ideal (90-95\%) transmission of the atmosphere. The atmospheric background noise is comparable with the quantum limit of the heterodyne receiver noise.

In Fig. 1 is an example of atmospheric transmission for SOFIA flying at $12 \mathrm{~km}$ altitude with the telescope elevation angle of 30 degree. The transmission has been calculated using Atmospheric Transmission at Microwaves (ATM) model [3]. Outside of relatively narrow absorption lines of the Earth atmosphere the transmission may be as good as $95-90 \%$, resulting in the atmospheric background noise temperature as low as $15-30 \mathrm{~K}$ for the most of $1 \mathrm{THz}$ band. The quantum limit of the noise of a Double Side Band (DSB) heterodyne receiver is about $\mathrm{T}_{\mathrm{N}}=h v / 2 k \approx 24 \mathrm{~K}$ at $1 \mathrm{THz}$ [4] and this noise level is comparable with the atmospheric background noise. Therefore the development of a heterodyne receiver approaching the quantum limit of sensitivity in $1 \mathrm{THz}$ band may substantially improve the capability of SOFIA, making possible to operate with nearly background-limited sensitivity. The goal of this work is to develop a low noise mixer for such receiver.

A mixer using quantum assisted tunneling in Superconductor-insulator-superconductor (SIS) junctions is the best solution for building a sensitive heterodyne receiver in $1 \mathrm{THz}$ band [5]. Earlier works on $1 \mathrm{THz}$ SIS mixer has been done using on-chip normal metal circuits and having a relatively narrow operation band [6], [7]. Recently a waveguide SIS mixer design with $\mathrm{Nb} / \mathrm{Al}-\mathrm{AlO} / \mathrm{Nb}$ junctions and $\mathrm{NbTiN}$ circuit has been explored with a big success in order to provide a 0.96-1.12 THz SIS receiver for Herschel Space Observatory (HSO) HIFI instrument [8]. In our work for CASIMIR project 
the goal is to develop a quasi-optical SIS mixer with a broader RF band centered at $1 \mathrm{THz}$.

\section{SIS MiXER DESIGN}

We developed a SIS mixer using high critical current density $\mathrm{Nb} / \mathrm{Al}$-AlN/NbTiN junctions with the normal state resistance to area product $\mathrm{R}_{\mathrm{N}} \mathrm{A}=6 \mathrm{Ohm} \mu \mathrm{m}^{2}$ [9]. The two SIS junction array is coupled to the double slot antenna as proposed in [10]. In contrast with the previous works we are using epitaxial $\mathrm{Nb}$ ground plane and a gold wire layer to form the matching circuit of the SIS mixer. The mixer microstrip circuit insulator is $\mathrm{SiO}$.

Due to a low resistivity of the epitaxial $\mathrm{Nb}$ film, approaching $0.2 \mu \mathrm{Ohm} \mathrm{cm}$ at $10 \mathrm{~K}$, the loss in the mixer circuit is relatively low. Another advantage of the design using epitaxial $\mathrm{Nb}$ ground plane instead of NbTiN is a better tolerance to the manufacturing errors. The $1 \mathrm{THz}$ frequency is well above the gap frequency of $\mathrm{Nb} \mathrm{F}_{\mathrm{gNb}}=700 \mathrm{GHz}$, and so at $1 \mathrm{THz}$ Niobium behaves as a normal metal. Therefore at $1 \mathrm{THz}$ a microstrip circuit made of $\mathrm{Nb}$ has no reduction of the speed of propagation of the signals related to the kinetic inductance. A manufacturing error in length or in positioning of the circuit parts is leading to an error in the phase length of the circuit elements. The error is smaller if $\mathrm{Nb}$ ground plane is used instead of NbTiN. In our design the substitution of NbTiN ground plane with $\mathrm{Nb}$ reduces the phase length of the micro-strip elements by about 1.6. This advantage may be useful in development of multibeam receivers, where a big number of identical mixers is required.

The mixer housing design is presented in Fig. 2(a). It is similar to one used in our work on $1.2 \mathrm{THz}$ SIS mixer [11]. The mixer housing components are: the base frame, the IF and DC bias board, and the IF and DC connectors. A Silicon lens with the mixer chip on it back side is fixed in a hole at the front plane of the mixer housing. The mixer chip layout is in Fig. 2(b). It is a twin SIS junction circuit coupled to a double-slot planar antenna. An electrical magnet is used to suppress the Josephson currents in SIS junctions.

The model prediction of the mixer on-chip coupling is presented in Fig. 3 (dashed line). For modeling we used the SUPERMIX software package [12]. The predicted mixer response is centered at $1 \mathrm{THz}$ and is expected to be about $250 \mathrm{GHz}$ wide. The central frequency of the measured FTS response matches the model prediction within 2\% (solid line in Fig. 3). The $3 \mathrm{~dB}$ bandwidth of the FTS response is close to the prediction, about $250 \mathrm{GHz}$ wide. An indentation in FTS response just above 1100 $\mathrm{GHz}$ is likely to be a trace of residual water vapor absorption around the $1163 \mathrm{GHz}$ line. In more details the mixer response is studied in heterodyne receiver experiment presented in the next section.

\section{EXPERIMENT AND DISCUSSION}

The test receiver used in our experiment comprises an Infrared Laboratories LH-3 cryostat, a solid-state local oscillator, and the bias electronics. The cryostat vacuum window is in Mylar $13 \mu \mathrm{m}$ thick. An infrared filter made of Zitex is located at the $77 \mathrm{~K}$ stage of the cryostat. The local oscillator power is coupled to the mixer beam using a Mylar beam splitter $13 \mu \mathrm{m}$ thick. The receiver intermediate frequency range is $4 \mathrm{GHz}-8 \mathrm{GHz}$ and the IF amplifier noise temperature is about $3 \mathrm{~K}$. An isolator is a)

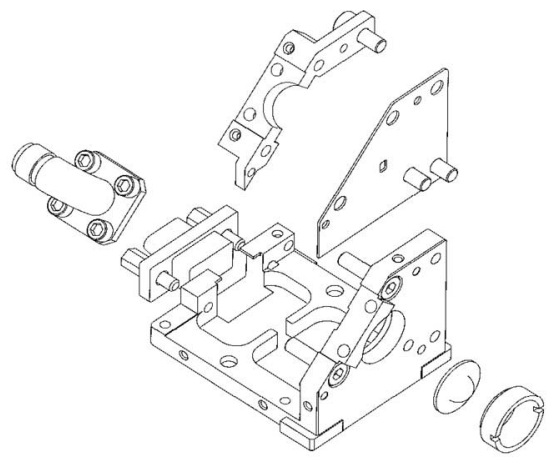

b)

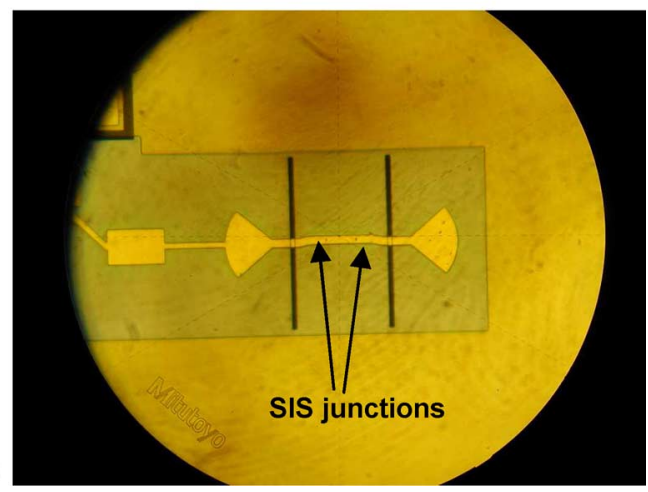

Fig. 2. (a) The mixer housing of a $1 \mathrm{THz}$ SIS mixer developed for SOFIA. It is similar to one used in our work on 1.2 THz SIS mixer [11]. The mixer housing consist of the base frame, IF and DC bias board, IF and DC connectors. A Silicon lens with the mixer chip on it back side is fixed in a hole at the front plane of the mixer housing. (b) The $1 \mathrm{THz}$ mixer chip layout. The mixer has a twin SIS junction circuit coupled to a double-slot antenna.

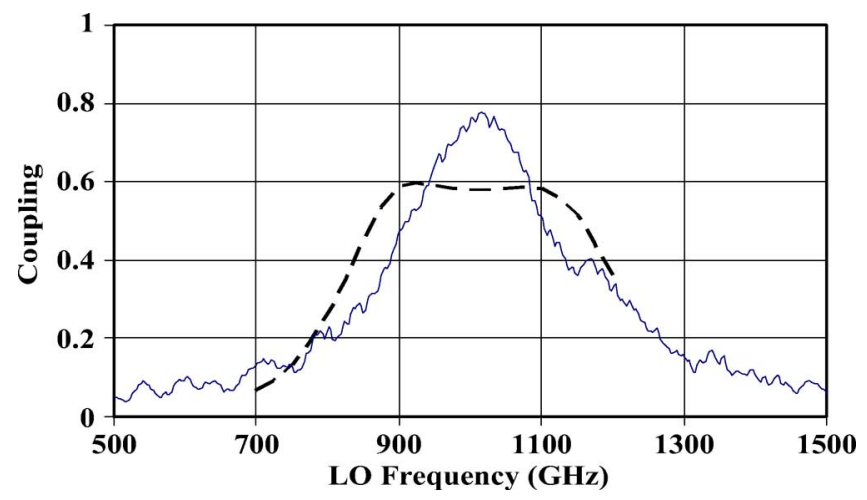

Fig. 3. The model prediction of the $1 \mathrm{THz}$ mixer on-chip coupling (dashed line) and the measured FTS response in arbitrary units (solid line). The central frequency of the measured FTS response matches the model prediction within $2 \%$.

used to avoid the mismatch between the mixer and IF amplifier. During the test the physical temperature at the mixer block was about $2 \mathrm{~K}$.

We used the Y-factor method for the measurement of the receiver double sideband noise. The ambient temperature and the liquid-nitrogen cooled loads are used as the reference signal sources. The minimum measured receiver noise temperature is $\mathrm{T}_{\mathrm{REC}}=353 \mathrm{~K}(\mathrm{Y}=1.50)$ at $924 \mathrm{GHz}$. The receiver noise corrected for the loss in the $13 \mu \mathrm{m}$ thick Mylar beam splitter is presented in Fig. 4. In a good agreement with the mixer design requirements the measured receiver bandwidth is about 250 $\mathrm{GHz}$ and is centered at $1 \mathrm{THz}$ frequency. The minimum receiver 


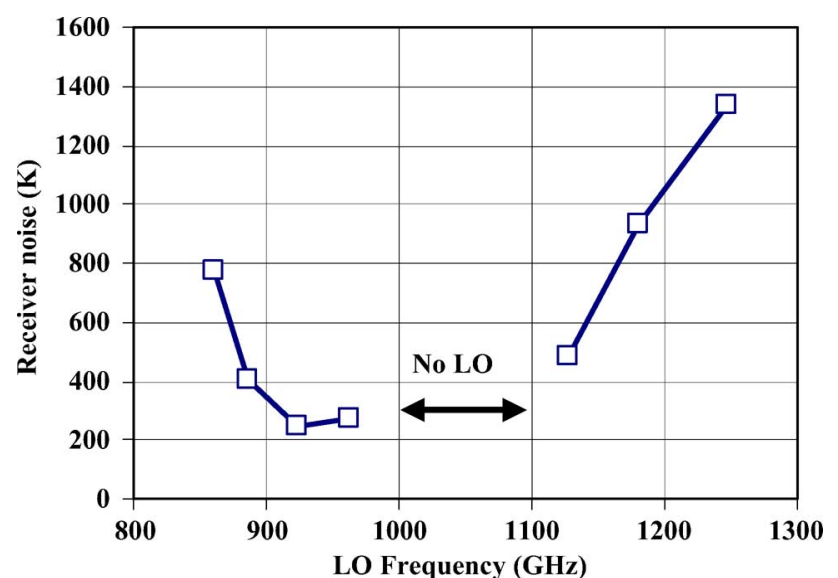

Fig. 4. The measured receiver noise corrected for the loss in the LO injection beam splitter. The receiver band is about $250 \mathrm{GHz}$ and it is centered at $1 \mathrm{THz}$. The break in the curve corresponds to the band with no LO available. The minimum receiver noise corrected for the loss in LO coupler is about $250 \mathrm{~K}$.

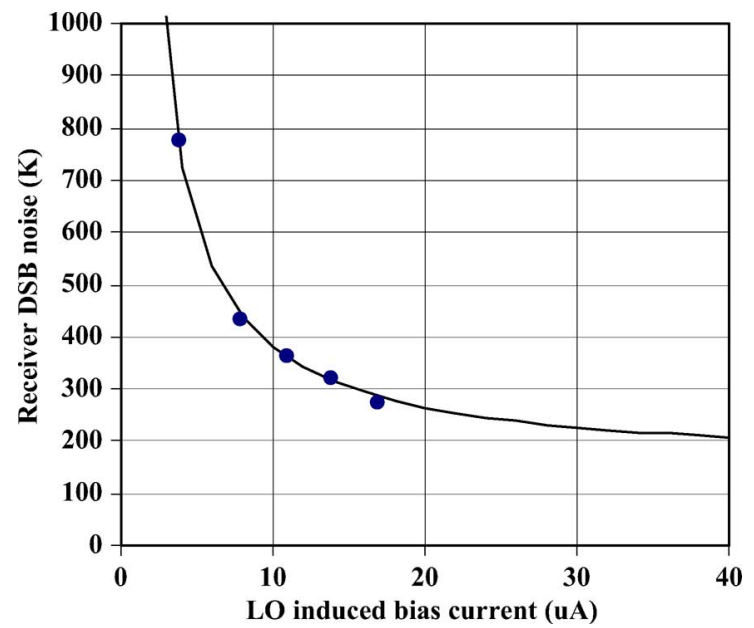

Fig. 5. The receiver noise as a function of the Local Oscillator induced DC current. The SIS junction bias voltage is fixed at V $=2.12 \mathrm{mV}$ and the $\mathrm{LO}$ frequency is $964 \mathrm{GHz}$. The receiver noise is corrected for the loss in the LO beam splitter. It is visible that the mixer noise may be improved at a higher level of LO power.

corrected noise temperature is $250 \mathrm{~K}$. We had no LO coverage for some part of the receiver band.

An example of the receiver performance as a function of LO power level is presented in Figs. 5 and 6. We are using the Local Oscillator induced DC current $\left(\mathrm{I}_{\mathrm{LO}}\right)$ as a measure of the LO power available at SIS junction in the mixer $\left(\mathrm{P}_{\mathrm{LO}}\right)$. It is known that in quasiparticle SIS mixer the $\mathrm{I}_{\mathrm{LO}}$ is proportional to the LO power $\mathrm{I}_{\mathrm{LO}}=e \mathrm{P}_{\mathrm{LO}} / h f[13]$ whre $e$ is the charge of an electron, $h$ is the Planck constant and $f$ is the frequency. The measurements are done at the varied levels of $\mathrm{P}_{\mathrm{LO}}$. The SIS junction bias voltage is fixed at $\mathrm{V}=2.12 \mathrm{mV}$ and the $\mathrm{LO}$ frequency is $964 \mathrm{GHz}$. The receiver noise is corrected for the loss in the $13 \mu \mathrm{m}$ Mylar beamsplitter.

As we can see in Fig. 5, the receiver noise is strongly dependent on the $\mathrm{P}_{\mathrm{LO}}$ and the level of available LO power is limiting the receiver sensitivity. It is also visible that the receiver noise may be improved at a higher level of LO power. The solid line is the fit to the measured receiver noise using (3).

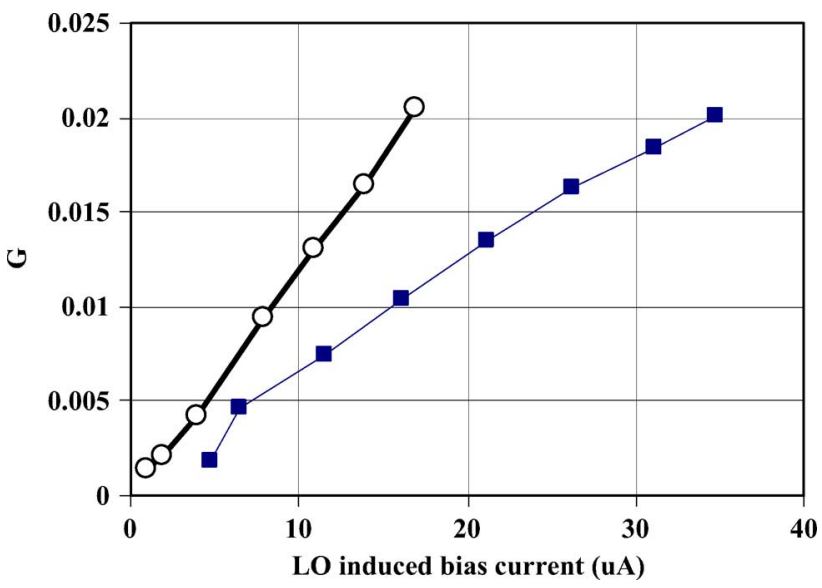

Fig. 6. Measured mixer conversion gain as a function of the local oscillator induced current $\mathrm{I}_{\mathrm{LO}}$. Here we are using the data measured at with $1 \mathrm{THz}$ mixer at $964 \mathrm{GHz}$ (open circles) and with a $1.2 \mathrm{THz}$ mixer design at $1128 \mathrm{GHz}$ (dots) from our previous work [11]. The SIS junctions are identical in the two mixers. The mixer gain is proportional $\mathrm{I}_{\mathrm{LO}}$ up to $35 \mu \mathrm{A}$.

Mixer conversion gain $\mathrm{G}$ is proportional to the difference in the receiver output power with room temperature $\mathrm{P}_{\mathrm{IF} \text { HOT }}$ and Nitrogen cooled loads $\mathrm{P}_{\mathrm{IF} \text { COLD }}$ measured in $\mathrm{Y}$ factor experiment: $\mathrm{G}=\gamma\left(\mathrm{P}_{\mathrm{IF} \text { HOT }}-\mathrm{P}_{\mathrm{IF} \text { COLD }}\right) / 2$. There the factor 2 denotes the fact that the conversion is performed in the two sidebands of a double sideband receiver. The coefficient $\gamma$ is determined through in-situ calibration of the IF amplifier chain using the SIS mixer shot noise as a reference signal. In the same calibration process we determined the IF chain equivalent noise temperature $\left(\mathrm{T}_{\mathrm{IF}}\right)$. The measured $\mathrm{T}_{\mathrm{IF}}$ is $3 \mathrm{~K}$ for the entire $4-8$ $\mathrm{GHz}$ band.

In Fig. 6 the measured mixer conversion gain is presented as a function of the local oscillator induced current $\mathrm{I}_{\mathrm{LO}}$. Here we are using the data measured at with $1 \mathrm{THz}$ mixer at $964 \mathrm{GHz}$ (open circles). The dependence $\mathrm{G}\left(\mathrm{I}_{\mathrm{LO}}\right)$ is linear up to the maximum available $\mathrm{LO}$ power at $\mathrm{I}_{\mathrm{LO}}=17 \mu \mathrm{A}$. To have an example of the operation with more LO power we give the data from our previous work [11] measured with $1.2 \mathrm{THz}$ mixer design at 1128 $\mathrm{GHz}$ (dots). The junction parameters in the two mixers are identical. At $1128 \mathrm{GHz}$ the mixer gain has been found proportional to the $\mathrm{LO}$ induced current up to $35 \mu \mathrm{A}$.

In the range of available LO power the measured conversion gain of the mixers is directly proportional to $\mathrm{I}_{\mathrm{LO}}$ :

$$
G_{M I X E R}=\beta I_{L O}
$$

where $\beta$ is a constant for each mixer. We can use this observation to make an estimation of the possible performance of the mixer when pumped to the optimal level of the $\mathrm{I}_{\mathrm{LO}}$ of about $35 \mu \mathrm{A}$ for our mixer.

It is known that the noise of SIS receiver is determined by mixer output noise temperature $\mathrm{T}_{\text {OUT MIXER, }} \mathrm{T}_{\mathrm{IF}}$, by the mixer conversion gain $\mathrm{G}$ and the front-end optics noise $\mathrm{T}_{\mathrm{OPT}}$ [14], [15]. For a double side band SIS mixer:

$\mathrm{T}_{\mathrm{REC}}=\mathrm{T}_{\mathrm{OPTICS}}+\left(\mathrm{T}_{\mathrm{IF}}+\mathrm{T}_{\text {OUT MIXER }}\right) / 2 / \mathrm{G}_{\mathrm{MIXER}}\left(\mathrm{P}_{\mathrm{LO}}\right)$ 


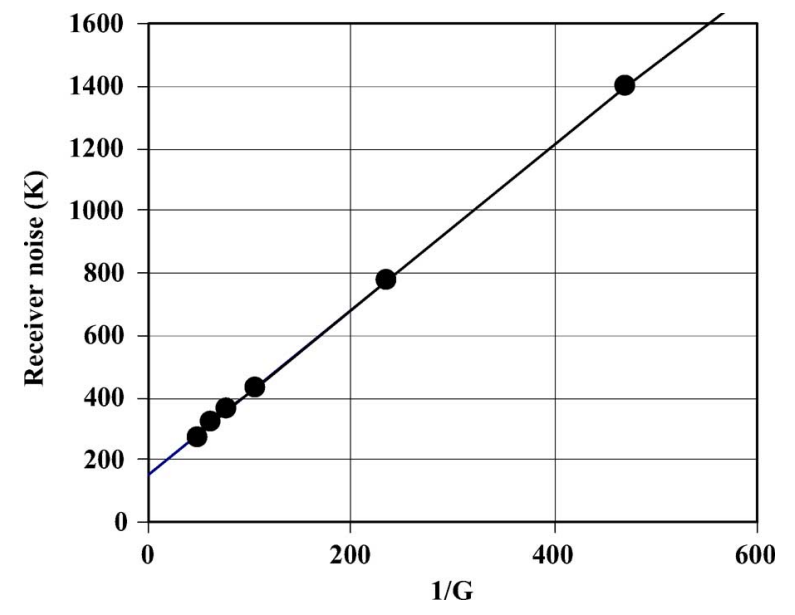

Fig. 7. SIS receiver noise versus conversion loss measured at $964 \mathrm{GHz}$. The receiver noise is corrected for the loss in the Local Oscillator coupler and the conversion loss is $1 / \mathrm{G}$, where $\mathrm{G}$ is receiver $\mathrm{SSB}$ conversion gain. The data points are obtained at the various levels of the Local Oscillator power. The measured data points are located at a strait line in a good agreement with the model introduced by [14], [15]. The line is a linear fit crossing the vertical axis at the level of $\mathrm{T}_{\mathrm{OPT}}=150 \mathrm{~K}$.

In this relation factor 2 is denoting the fact that down conversion is performed in the two mixer sidebands. The validity (2) for our mixer is confirmed by the data in Fig. 7. There the receiver noise is plotted as a function of the conversion loss $(1 / \mathrm{G})$. As expected the data points are located at a strait line. The line is crossing the $T_{\text {REC }}$ axis at the level of the receiver front-end optics noise $\mathrm{T}_{\mathrm{OPT}}=150 \mathrm{~K}$.

Substituting (1) in (2) we are getting:

$$
\mathrm{T}_{\mathrm{REC}}=\mathrm{T}_{\mathrm{OPTICS}}+\left(\mathrm{T}_{\mathrm{IF}}+\mathrm{T}_{\mathrm{OUT} \text { MIXER }}\right) / 2 /\left(\beta \mathrm{I}_{\mathrm{LO}}\right)
$$

where $\mathrm{T}_{\text {OPTICS }}$ is the noise contribution of the receiver optics, $\mathrm{T}_{\mathrm{IF}}$ is the noise of the IF chain, and $\mathrm{T}_{\text {OUT MIXER }}$ is the noise at the mixer IF port. This relation is used to make extrapolation of the receiver noise data in Fig. 5. The receiver noise may be close to $220 \mathrm{~K}$ using 2 times more LO power than available in our experiment. We will verify this estimate in a future work on the practical astronomical receiver.

\section{CONCLUSION}

We developed a broad-band low noise SIS mixer for $1 \mathrm{THz}$ channel of CASIMIR instrument of Stratospheric Observatory for Far Infrared Astronomy (SOFIA). The mixer band of operation is $0.875-1.125 \mathrm{THz}$, about $250 \mathrm{GHz}$ wide. The minimum DSB receiver noise measured in our test receiver is 353 $\mathrm{K}(\mathrm{Y}=1.50)$. The receiver noise corrected for the loss in the LO injection beam splitter is $250 \mathrm{~K}$. The Local Oscillator power level in our test was below an optimal level, and the mixer performance may be improved using more LO power. The minimum DSB receiver noise in optimized receiver with more LO power available may be as low as $220 \mathrm{~K}$.

The developed mixer appears to be a prospective element for construction of a low-noise heterodyne receiver for SOFIA.

\section{ACKNOWLEDGMENT}

The authors thank Imran Mehdi (JPL) for the loan of $1 \mathrm{THz}$ local oscillator.

\section{REFERENCES}

[1] E. E. Becklin, "Stratospheric Observatory For Infrared Astronomy (SOFIA)," in Space Telescopes and Instrumetst, Proc. of SPIE, Mar. 1998, vol. 3356, pp. 492-499, 1998.

[2] M. L. Edgar and J. Zmuidzinas, "CASIMIR: A submillimeter heterodyne spectrometer for SOFIA," in Airborne Telescope Systems, Proc. SPIE, 2000, vol. 4014, pp. 31-42.

[3] J. R. Pardo, J. Cernicharo, and E. Serabyn, "Atmospheric Transmission at Microwaves (ATM): An improved model for $\mathrm{mm} / \mathrm{submm}$ applications," IEEE Trans. Antennas and Propagation, vol. 49/12, pp. 1683-1694, 2001.

[4] A. R. Kerr, M. J. Feldman, and S.-K. Pan, Receiver Noise Temperature, the Quantum Noise Limit, and the Role of the Zero-Point Fluctuations NRAO Electronics division internal report, No 304, September 1996.

[5] J. Zmuidzinas and P. L. Richards, "Superconducting detectors and mixers for millimeter and submillimeter astrophysics," Proceedings of the IEEE, vol. 92, no. 10, pp. 1597-1616, 2004.

[6] M. Bin, M. C. Gaidis, J. Zmuidzinas, T. G. Phillips, and H. G. Leduc, "Quasi-Optical SIS mixers with normal-metal tuning structures," IEEE Trans. Applied Superconductivity, vol. 7, no. 2, pt. 3, pp. 3584-3588, Jun. 1997.

[7] S. V. Shitov, B. D. Jackson, A. M. Baryshev, A. V. Markov, N. N. Iosad, J.-R. Gao, and T. M. Klapwijk, "A low-noise double-dipole antenna SIS mixer at 1 THz," Physica C, vol. 372, pp. 374-377, 2002.

[8] B. D. Jackson, G. de Lange, T. Zijlstra, M. Kroug, J. W. Kooi, J. A. Stern, and T. M. Klapwijk, "Low-noise 0.8-0.96- and 0.96-1.12-THz superconductor-insulator-superconductor mixers for the Herschel space observatory," IEEE Trans. Microwave Theory and Techniques, vol. 54, pp. 547-558, Feb. 2006.

[9] B. Bumble, H. G. LeDuc, J. A. Stern, and K. G. Megerian, "Fabrication of Nb/Al-Nx/NbTiN junctions for SIS mixer applications," IEEE Trans. Applied Superconductivity, vol. 11, no. 1, pp. 76-79, 2001.

[10] J. Zmuidzinas, H. G. Leduc, J. A. Stern, and S. R. Cypher, "Two-junction tuning circuits for submillimeter SIS mixers," IEEE Trans. Microwave Theory and Techniques, vol. MTT-42, no. 4, pp. 698-706, Apr. 1994.

[11] A. Karpov, D. Miller, F. Rice, J. A. Stern, B. Bumble, H. G. LeDuc, and J. Zmuidzinas, "Low noise SIS mixer for far infrared radio astronomy," in Millimeter and Submillimeter Detectors for Astronomy II, Proceedings of SPIE, October 2004, vol. 5498, pp. 616-621.

[12] J. Ward, F. Rice, G. Chattopadhyay, and J. Zmuidzinas, "Supermix," in Proc. of the 10th Int. Symp. on Space THz Tech., March 16-18, 1999, pp. $268-280$.

[13] J. R. Tucker and M. J. Feldman, "Quantum detection at millimeter wavelengths," Reviews of Modern Physics, vol. 57, no. 4, pp. 1055-1113, Oct. 1985.

[14] R. Blundell, R. E. Miller, and K. H. Gundlach, "Understanding noise in SIS receivers," Int. J. IR and MM Waves, vol. 13, no. 1, pp. 3-26, Jan. 1992.

[15] Q. Ke and M. J. Feldman, "A technique for noise measurements of SIS receivers," IEEE Trans. Microwave Theory Tech., vol. 42, no. 4, pp. 752-755, Apr. 1994. 